\title{
Schreiben von Hrn. Dr. F. Terby betr. den weissen Fleck auf dem Saturnsring.*)
}

Monsieur le Docteur Kreutz ayant bien voulu me communiquer immédiatement le no. d'avril du sidereal Messengers, j'ai pu prendre connaissance des observations de la région blanche sur l'anneau de Saturne faites en Amérique par MM. Brashear, Very, Swift, Brooks et Payne.

MM. Brashear, Very et Payne attribuent plutốt à un phénomene de contraste ce qu'ils ont observé et MM. Swift et Brooks croient avoir vu réllement la région blanche que j'ai signalée.

Il existe, en effet, sur l'anneau, contre l'ombre du globe, une apparence que l'on est porté à attribuer au contraste; il est étonnant que l'on semble s'en être si peu occupé jusqu'ici: c'est généralement une bande étroite, blanchâtre, à bord bien défini, qui longe l'ombre et qui paraît d'éclat très-variable; ne serait-on pas fondé à se demander si cette variabilité et cette netteté du bord se concilient bien avec l'hypothèse d'un effet de contraste ? Quoiqu'il en soit, j'ai remarqué très-souvent ce détail et je dois déclarer que le phénomène des 6 et 12 mars ne lui était point comparable tant son intensité était grande; de plus il s'étendait plus loin que cette bande et avait une

*) Vergl. die spätere Notiz von Dr. Dreyer in A. N. 2894. limite mal définie, comparable en cela aux régions d'éclat blanc et vif que l'on remarque sur Jupiter. La petite zone attribuée au contraste ne s'aperçoit que moyennant une attention spéciale, elle échappe même souvent à l'observation, tandisque la région blanche des 6 et 12 mars était a ce point brillante, blanche et étendue, que le restant de l'anneau si brillant, notamment dans la région $B$, prenait un aspect terne et presque grisâtre en sa présence.

Je n'oserais me prononcer sur la nature exacte des phénomènes observés par les astronomes américains: tandisque MM. Brashear, Very et Payne me semblent n'avoir observé que la blancheur attribuée à tort ou à raison au contraste, MM. Swift et Brooks pourraient bien avoir observé un phénomène identique avec celui des 6 et 12 mars, quoique d'un éclat moins remarquable. Mais les diverses observations faites ne seront comparables à celles de Louvain que lorsque nous connaîtrons les instants précis auxquels les phénomènes ont été constatés.

Je n'ai encore appris aucun détail de l'observation faite par Monsieur Mc Leod.

Louvain le 16 avril 1889.

Dr. F. Terby.

\section{Oppositions-Ephemeriden der Planeten}

Wegen der sehr starken Annäherung der Bahnen dieser beiden Planeten an einander und einer schon 1870 von K. v. Littrow (Denkschriften der Wiener Akad. XXXI.r 83) angezeigten nur um wenige Tage verschiedenen Durchgangszeit derselben durch diese Bahnnähe im Sept. 1888 , ist im Jahre vorher diese Proximität von meinem Sohne auf neueren Grundlagen genauer berechnet worden (A. N. 118.77), zugleich mit Ephemeriden für die im März und April 1888 stattgehabten Oppositionen, welche in dem Berl. Jahrbuche für 1890 enthalten sind. Beobachtungen dieser Oppositionen sind mir bisher nur aus Paris bekannt geworden, wo Herr Callandreau folgende Abweichungen der Rechnung von der Beobachtung gefunden hat (C. R. CVII.888) im Sinne B-R :

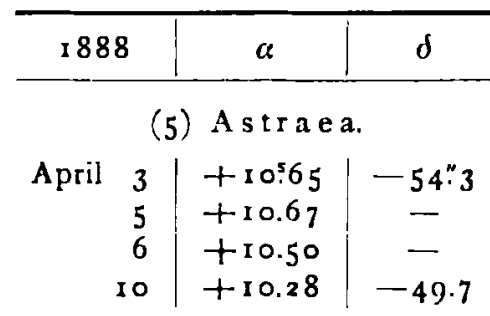

(8) Flora.

$$
\begin{array}{r|r|r}
\text { April } 6 & +9.73 & -51.1 \\
14 & +8.61 & -50.4 \\
16 & +8.48 & -47.9
\end{array}
$$

Obgleich nun die angeführte Berechnung der um den 9. Sept. v. J. stattgehabten kleinsten Entfernung der beiden Planeten diese Entfernung nicht so klein ergeben hat, um

\section{(5) Astraea und (8) Flora für 1889.}

eine merkliche Einwirkung derselben auf einander erwarten zu lassen (dieselbe betrug etwa 0.04, während die Bahnen sich bis auf 0.006 nähern), so schien doch die Unsicherheit der Elemente der Planeten eine noch etwas grössere Annäherung nicht auszuschliessen. Inzwischen haben gegenwärtig die obigen Beobachtungen gezeigt, dass bei beiden Planeten der Ephemeriden-Fehler annähernd derselbe, die Verschiebung des Ortes daher bei beiden in demselben Sinne anzunehmen ist, und die berechnete 4 tägige Zwischenzeit zwischen dem Durchgange des einen und des andern durch die Bahnnähe nicht erheblich von dem thatsächlichen Hergange abweichen wird. Dennoch möchte eine erneute Beobachtung beider Planeten auch in diesem Jahre erwiunscht sein, um über die Grenzen der Einwirkung dieser Himmelskörper auf einander einige bestimmte Feststellungen zu gewinnen. Ich habe daher Herrn Stud. astr. Stelzer hier in Breslau veranlasst, die nachstehende Ephemeride der Astraea (nach Elementen von Dr. Neugebauer) zu berechnen, während die für Flora wiederum von meinem Sohne nach den Brünnow'schen Tafeln gerechnet ist. - Der Tafel-Fehler der Flora ist, wie die obigen Pariser Beobachtungen zeigen, nicht unbeträchtlich, hat sich jedoch seit 3 Jahren nicht erheblich vergrössert, wie man aus den in A. N. 114.38 enthaltenen (für etwa gleiche Entfernung von der Erde geltenden) Beobachtungen von Herrn Pomerantzeff in Taschkent ersieht. Es dürften daher bei Beobachtungen im gegenwärtigen Jahre schon die Vergleichungen der EphemeridenFehler von einer etwaigen aussergewöhnlichen Einwirkung eine Andeutung geben, auch noch vor Ausführung von Elementen-Verbesserungen, wie sie für beide Planeten erforderlich erscheinen. 